\title{
Supply Chain Network Design and Tactical Planning in the Dimension Stone Industry
}

\author{
Gangaraju Vanteddu \\ Department of Marketing, Harrison College of Business \& Computing, \\ Southeast Missouri State University, Cape Girardeau, USA \\ E-mail: gvanteddu@ semo.edu (Corresponding Author) \\ Gillian M. Nicholls \\ Department of Marketing, Harrison College of Business \& Computing, \\ Southeast Missouri State University, Cape Girardeau, USA \\ Email: gnicholls@semo.edu
}

\begin{abstract}
A dimension stone supply chain has certain unique characteristics despite having many similarities to a typical manufacturing/processing industry supply chain. Examples of these characteristics include the multitude of dimension stone varieties dispersed over large geographical regions, variable raw dimension stone sizes due to the varying rock deposit quality, and variability in physical stone properties among different varieties. The hardness, texture, fracture, cleavage, and chemical composition vary between dimension stones of different varieties. These unique characteristics produce multidimensional complexity in modelling different processes from the rock excavation stage to the retail store stage. In this research, a dimension stone supply chain is analyzed from strategic and tactical perspectives that address the issues of supply chain network design and production planning. This research reflects the specific characteristics of a dimension stone supply chain that differentiate it from other mineral commodity supply chains. A Mixed Integer Linear Programming (MILP) based formulation is proposed to model the capacitated facility location problem to aid in the supply chain network design, and a linear programming-based model is proposed to address material transformation related supply chain process issues to facilitate medium-term tactical planning. Detailed numerical analyses are also presented to illustrate the proposed mathematical models.
\end{abstract}

Keywords: dimension stone supply chain, network design, mixed integer linear programming, capacitated facility location

\section{INTRODUCTION}

A dimension stone is a naturally produced rock such as granite, marble, sandstone, and slate that is mined primarily for use in construction for structural and/or ornamental purposes. The composition of different types of rocks typically consists of one or more minerals depending on the origin of their occurrence. Based on their geological origin, the three most commonly used rocks in the dimension stone industry are Igneous, Sedimentary, and Metamorphic varieties (USGS, 2017). Igneous rocks such as granite, quartz, and basalt among others result from volcanic eruptions. Sedimentary rocks such as sandstone and shale are the end products of sedimentation processes over millions of years. Metamorphic rocks such as marble, slate, quartzite, etc. result from the intense transformation processes that pre-existing rocks such as igneous and sedimentary undergo within the bowels of the earth. Granite, marble, limestone, sandstone, and slate are some of the popular varieties of dimension stones commonly used across the globe.

Granite and marble are the most popular varieties of dimension stones. Worldwide production of granite and marble was approximately 142 million tons in 2013 per Italy's Internazionale Marmi e Macchine Carrara S.p.A with the top five producing countries in descending order by tonnage being China, Turkey, India, Iran, and Italy. These countries account for about $72 \%$ of the worldwide production (USGS, 2016). USGS also estimates that consumption of dimension stone in the United States for the year 2015 (the latest data currently available) was approximately US $\$ 2.76$ billion.

Despite its inherent supply chain complexity from the raw stone supplier stage to the consumer stage the dimension stone industry has not benefitted from the path breaking advances in the operations research area. Compared to other mineral commodity supply chains such as coal, crude oil and iron ore, dimension stone supply chains are relatively more intricate having to deal with many constraints at all the stages. A mineral commodity supply chain typically handles a very small set of specific outputs, whereas in the dimension stone industry one must contend with a wide assortment of colors, textures, and patterns in each of the many stone varieties. The stone variety makes the design of the relevant supply chain network that much harder. For example, there are about 300 varieties of granite alone currently available in the world market (Government of India: Indian Bureau of Mines, 2014). This is further compounded by the dispersed location of the quarry excavation sites, complexity of processing operations, and highly variable domestic and external demand for different dimension stone products, such as slabs and tiles of different thicknesses. Most of the dimension stone extraction operations are concentrated in developing countries with poorly developed supply chain networks, which further add to the complexity of the supply chain design and relevant tactical planning aspects. Probable lack of familiarity or awareness of supply chain philosophy 
and the relevant quantitative models for maximizing the supply chain surplus hinder the adoption of supply chain best practices in the dimension stone industry as evidenced by the scant research literature available in this area.

Dispersed locations of the dimension stone excavation sites (quarries), processing facilities, and markets pose several challenges in designing the supply chain network at the strategic level. Naturally occurring faults and cracks in the rock formations make raw dimension stone sizes highly variable thereby making logistics and production planning complicated and expensive. For example, variability in the raw dimension block size makes it difficult to plan for Less than Truckload (LTL) or Full Truckload (FTL) shipments, which in turn impact inbound and outbound transportation costs. Raw block size dimensions also affect the optimal allocation of the blocks onto a variety of processing equipment, because certain overhead expenses remain constant irrespective of the size of the raw dimension block. Sometimes, unavailability of raw blocks of appropriate dimensions may force the relevant supply chains to plan for substitute supply sources for a particular variety elsewhere (with potential loss of revenue for inferior substitute colors) to satisfy the demand. Also, once the supply chain architecture is finalized, optimal planning of finishing operations (cutting, polishing, trimming, etc.) at the processing facilities is also impacted by the physical properties (hardness, cleavage, fracture, specific gravity, etc.) of the raw dimension stones making it harder to come up with optimal tactical plans to execute various raw stone transformation processes. For example, granite varieties with more quartz content (\# 7 on Mohs' hardness scale) in their composition tend to be much harder to process compared to granite varieties with more Orthoclase Feldspar content (\# 6 on Mohs' hardness scale).

Supply chain network design issues involving the determination of the location and capacity of the processing facilities and/or warehouses at the strategic level and the tactical planning issues that facilitate the development of the relevant rough-cut capacity plans given a specific supply chain network have not attracted adequate attention in literature particularly in the context of the dimension stone industry. Despite extensive search across research databases and open platforms, the authors have found very little research literature that explores the supply chain aspects of the dimension stone industry. Saetta and Tiacci (2005) proposed developing a lean simulation model to manage the supply chain for the natural stone industry. They indicated what the primary difficulties in using this approach might be vs. the potential benefits. Bakhtavar et al. (2017) developed a fuzzy-weighted binary integer goal programming model to identify the best site at which to build a central processing plant for a set of small nearby dimension stone quarries. The site selected by the model had the shortest distance to electrical power and staffing; shortest distance to the quarries on average; and the least expensive land acquisition cost.

Even though there is a gap in the research concerning the strategic and tactical aspects of dimension stone supply chains, there is some research available on the upstream quarry operational aspects and other extraction industries. For example, McKenzie et al. (2008) in their research propose a shortest-path algorithm to determine the optimal movement of the feeder - machinery utilized to transfer the aggregate mixture (sand and gravel) onto a conveyor belt - to the mining frontier and to minimize the loader cycle-time costs. Newman et al. (2010) provide a thorough review of optimization models used in the planning and design of mines, production scheduling, equipment selection etc. Careddu et al. (2017) take a marketing perspective in their research exploring the reasons for the success of marble and the failure of granite excavated in the Sardinia region of Italy by focusing upon the commercial development, product portfolio, quality, and customer service aspects. Safari et al. (2010) used Analytic Hierarchy Process (AHP) to select a location for a mineral processing plant close to an iron ore mine by considering factors including distance from plant to mine, heavy machinery transport access, the quantity of excavation required and distance from plant to the tailing dam and waste sites. Goodfellow and Dimitrakopoulos (2017) propose a two-stage stochastic Mixed Integer Nonlinear Programming (MINLP) method to model extraction of minerals, transforming into products, and transportation to customers. The transformation of the minerals included separation of ores, blending, or further processing. The goal was to maximize the mining firm's net present value as products were delivered to customers or the spot market.

Application of Mixed Integer Linear Programming (MILP) models in supply chain network design and tactical and operational planning is a relatively recent phenomenon with applications in a wide variety of supply chains. In one of the earliest attempts, Arntzen et al. (1995) evaluate a Global Supply Chain Model (GSCM) for Digital Equipment Corporation's supply chain network. A GSCM is a large MILP based supply chain network design model to minimize cost and/or weighted cumulative production and distribution times subject to a variety of supply chain network related constraints. Korpela and Lehmusvaara (1999) present a warehouse network design model that takes into consideration both qualitative and quantitative aspects. They propose an Analytic Hierarchy Process (AHP) and MILP based model for maximizing overall logistics service performance while reflecting customer-specific logistics service requirements and other network related limitations. Malek and Rahim (2017) identified critical success factors in supply chain management for assemble to order (ATO) companies which included proper material classification, strategies for transforming raw materials, and the quality of relationships with suppliers. This has implications for the dimension stone industry in which stones of a specified size and thickness are shaped to order. Kallrath (2002) presents a simultaneous strategic and operational planning model for a chemical industry to maximize net profit with a non-linear price structure for the raw material purchase model. Thanh et al. (2008) also consider a MILP based model to address strategic and tactical decisions in their proposed productiondistribution supply chain network model with deterministic demands. Their model was designed with the flexibility for decision variables to be dynamic over a given planning horizon. Tsiakis and Papageorgiou (2008) present a MILP based supply chain network model of their production and distribution network by considering operational limitations related to quality, production, supply constraints, and financial limitations. The financial limitations include production costs, transportation costs, external duties, etc. In another study, Gumus et al. (2009) address the issue of optimal product flow between the factories, warehouses, and 
distributors in the context of a multi-national company alcohol-free beverage maker. They propose a neuro-fuzzy and MILP based approach to facilitate the supply chain network design. Corsano and Montagna (2011) propose a MILP based supply-chain network design model that also considers simultaneously operational and planning decisions to aid with the optimal allocation of storage tanks in a batch process industry. Sabzevari Zadeh et al. (2014) designed a steel supply chain network using two separate models with one using a MILP approach and the other a MINLP approach. A set of numerical tests were run using a commercial solver, and the MILP performed the best for medium and large-scale problems based upon computational time.

With the goal of improving the responsiveness of a supply chain, Babazadeh et al. (2013) present a MILP based supply chain network design model by primarily considering outsourcing, flexibility, transportation modes, and crossdocking aspects among others. In the context of a nonmanufacturing supply chain network design, Etemadnia et al. (2015) propose a MILP based fruit and vegetables supply chain network design model. The model was designed to minimize the costs of locating wholesale facilities and the network transportation costs to support establishing costefficient connections between production sites and consumption markets. Sharifzadeh et al. (2015) use a MILP formulation to optimize the biofuel supply chain design and operations under uncertainty. Fattahi et al. (2016) present a multi- commodity supply chain network design MILP model to facilitate strategic and tactical planning. Their model maximizes the net income of the supply chain by considering different interconnected time periods and capacity options subjected to investment related budgetary constraints.

There is a potential for improving the financial performance of firms in the dimension stone industry by better integration of their supply chains. Khan and Wisner (2019) found that while there was not a significant relationship between supply chain agility and performance there was an indirect connection through internal learning processes. Baud-Lavigne et al. (2016) present a MILP model for addressing simultaneously a joint product-family and supply chain design problem by considering facility, distribution center locations, and supplier options. Boujelben et al. (2016) present a multi-period facility location problem under multiple operational constraints solved using a twophase solution approach, wherein the outputs of the first phase formulated as a set-partitioning problem are used as inputs for the second phase facility location MILP.

The typical architecture of dimension stone supply chains and the constraints under which they operate as primarily witnessed in the Indian subcontinent region by one of the authors afford excellent opportunities for the application of optimization techniques. These opportunities include making long-term (strategic) decisions such as determining the location of the processing facilities, warehouses etc.; medium-term (tactical) decisions such as determining the rough-cut capacity plans, master production schedules etc.; and operational (short term) decisions. The large number of varieties in any one type of dimension stone results in different processing requirements and given continuously changing customer preferences for different varieties, it is not practical to combine tactical planning related aspects with the strategic network design. Thus, two different models have been proposed in this research to address the unique aspects of strategic and tactical planning in the dimension stone industry.

This research considers a typical dimension stone supply chain from the raw stone quarrying stage to the distribution stage. A MILP model is proposed to determine the processing facility location(s) and the relevant capacity allocation(s) at the strategic level. Because of fragmentation of demand at the retailer stage for exported material, the retailer stage is explicitly not considered in our model, and the research scope is limited to the supply chain contained within a single nation. Thus, the last stage of the supply chain in our model will be the location of distribution centers/warehouses (typically cities or major population centers) for fulfilling the domestic demand and the relevant seaports to fulfill the external demand. Decoupling of the retail stage would not have any significant effect on the optimal solution to the model presented in this research given the architecture of a typical dimension stone supply chain, which tends to be additive.

An illustrative example for a dimension stone supply chain network design in a developing nation is also provided to facilitate the understanding and use of the proposed model by industry practitioners. In addition, to address the capacity planning/allocation issues and facilitate the development of a master production schedule at the processing facility stage of a dimension stone supply chain, a linear programming model is proposed for developing tactical production plans in the medium term. This research has focused on a typical granite supply chain in one of the major supplier nations, India, but the proposed models are generic enough to be used for any dimension stone supply chain in any part of the world. The proposed network design model and the tactical production-planning model could be used independently by industry practitioners depending on whether or not they already have a processing facility. However, combining these two models in this research is believed to be particularly useful for practitioners in small and medium scale granite industries in developing countries. Such practitioners would typically be interested in knowing the approximate costs for potential tactical production plans in addition to knowing the optimal processing facility location to perform fund flow analyses, estimate working capital requirements etc. The proposed models have potential applications for a separate industry as only minor modifications would be required to use them to optimize a ceramic tile supply chain network design as well.

The rest of the paper is structured as follows, a typical dimension stone supply chain network is described in section 2 , a strategic supply chain network design model is proposed in section 3 to address processing facility location and capacity allocation issues, and section 3 also contains a tactical production-planning model for a dimension stone processing facility. In section 4 illustrative numerical examples are presented for the theoretical models presented in section 3. Discussion of the research highlights are presented in section 5. Finally, conclusions and future research directions are presented in section 6 . 


\section{DESCRIPTION OF A DIMENSION STONE SUPPLY CHAIN NETWORK}

A typical dimension stone supply chain consists of three primary processes as shown in Figure 1. The processes are: raw stone excavation and inbound transportation to the processing facility; raw stone processing operations (primarily cutting, polishing, and trimming) performed at the processing facility; and packaging and outbound transportation of the processed slabs and tiles to either domestic distribution centers or to the nearest seaport for export.

For a typical representation of dimension stone supply chain network, please see Figure 2. This figure depicts the flow of product from quarries to processing facilities and on to distribution points. Figures $\mathbf{1}$ and $\mathbf{2}$ are based on one author's previous work experience in a managerial capacity within the granite industry. The supply chain begins with confirmed and forecasted demand for different varieties of granite of varying thicknesses. Supply orders from domestic distribution centers and external export destinations are placed with either their own quarries or the prospective raw stone suppliers. Simultaneously purchase orders will be placed for consumables that are used in quarry excavation operations like gelatin sticks, electric detonators, ordinary detonators, different sizes of drill bits, etc. Other purchase orders are placed for maintenance, repair and operations (MRO) supplies used in the processing of the raw blocks of stone such as steel grit (also known as steel shots), reciprocating gang saw blades, lime, diamond wire saws, etc.

Rough-cut granite blocks are extracted through controlled explosions of the granite deposit. Electrical detonators and gelatin sticks are placed in pre-drilled holes within the sheet rock deposit and detonated carefully in a closely monitored operation to extract the rough-cut sections. Rough-cut granite blocks come in different sizes depending on the variety and the natural defects in the sheet rock. Once the rough-cut block reaches the processing facility, it primarily undergoes three major transformation operations: cutting, polishing, and trimming.

During the cutting operation, a raw block is cut into slabs of different thicknesses depending on the final use and customer requirements. For example, slabs of $2-3 \mathrm{~cm}$ thickness are typically used for making kitchen countertops, and $1 \mathrm{~cm}$ thick slabs are typically used as flooring tiles. For granite slabs of thickness $5 \mathrm{~cm}$ or more, raw blocks undergo a process called slabbing, wherein circular saws with metal segments that contain artificial diamond particles are deployed to cut the stone into slabs with running water acting as a coolant to prevent overheating. The process of cutting granite slabs less than $5 \mathrm{~cm}$ in thickness uses large reciprocating gang saws with steel blades as the cutting medium, steel grit as an abrasive, and water as a coolant. Steel blades are typically under tension to prevent them from buckling during the operation. These gang saws can process approximately $80-100 \mathrm{sqft} / \mathrm{hr}$. High-pressure water jets and diamond wire saws are used for the cutting operation in more modern settings. The continuous reciprocating motion of the steel saws with steel grit and coolant mixture circulated over the body of the raw block creates an abrasive action where the steel grit between the reciprocating steel blades and the raw stone cuts the stone.

Sawn slabs are then sent to the polishing section of the processing plant to grind the rough grain surfaces to a glossy or semi-glossy finish. Depending on the customer requirements, sometimes sawn slabs undergo sandblasting or flaming for rough finishes as opposed to polishing to a smooth finish. Modern polishing processes typically consist of line polishers with several polishing heads (typically 16) arranged in sequence. Each polishing head will typically have 6 polishing bricks to grind the rough grains of the slab surface through abrasive action. In areas where labor costs are cheaper, such as in developing countries, 4-Head or 2Head mechanized or manual polishers are used for polishing the slab surface instead of line polishers. Polishing brick grains are coarser or finer depending on the type of the granite, its texture, its relative hardness, and the customer requirements. Typical polishing operation output is in the range of 5-10 sqft/minute.

After the polishing operation is completed, the slabs undergo trimming operations to achieve the specified dimensions as requested by the customer. Subsequently, the slabs are packaged for transportation. Then slabs are sent to the nearest seaport to satisfy the external demand or to the domestic distribution centers to satisfy domestic demand.

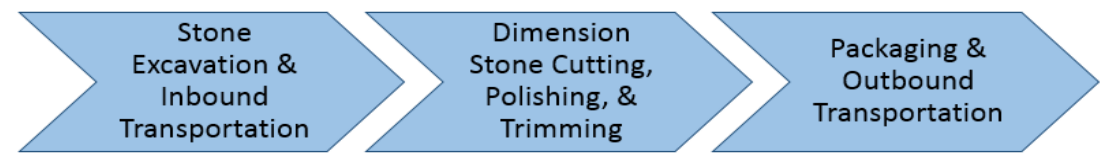

Figure 1 Process perspective of a dimension stone supply chain

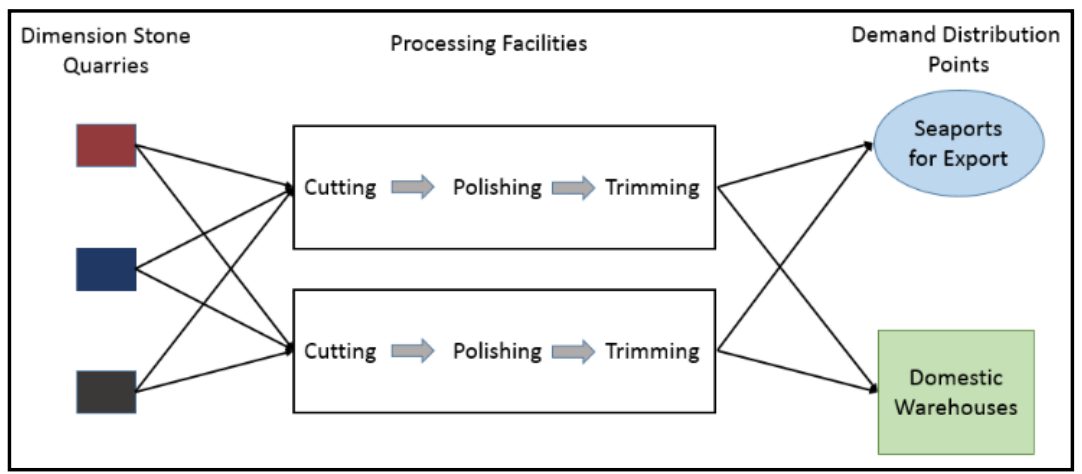

Figure 2 A typical representation of a dimension stone supply chain 
3. SUPPLY CHAIN NETWORK DESIGN AND TACTICAL PLANNING

\subsection{Key Elements of a Dimension Stone Supply Chain}

\subsubsection{Dimension Stone Supply Chain Processes}

Supply chain network design to determine the optimal location of the processing facilities and the relevant capacity allocations to minimize the total cost of processing and transportation presents certain unique challenges in the dimension stone industry. At the strategic level, a dimension stone supply chain network primarily consists of three processes, inbound transportation (quarries to processing facilities), raw block conversion (raw blocks to slabs/tiles transformation at the processing facility) and outbound transportation (from processing facilities to domestic distribution centers and/or seaports for export). Key elements of all three processes are briefly described below. Inventory costs are not considered explicitly in this model as typically orders are processed and satisfied only after receiving the firm orders (because of highly variable customer preferences) for specific varieties and thicknesses. As a result, quantities processed in each time period are consumed in the same time period with backlogs normally not entertained.

\subsubsection{Inbound Transportation}

Inbound transportation costs from quarries to the processing facilities depend on the size of the rough-cut raw blocks as Full Truck load (FTL) vs. Less Than Truck load (LTL), and the capacity of handling facilities at the unloading point (High capacity vs. Low capacity). Certain varieties of granites typically come in larger sizes as in the case of red varieties from the Indian sub-continent region compared to others such as the black varieties, which come in relatively small sizes because of naturally occurring cracks and other defects. The block size variations result in different costs of handling and transportation. Typical FTL costs charged for transporting larger raw blocks cost less than LTL costs for transporting smaller sized blocks based on unit cost of transportation per square foot. Likewise, raw blocks to be processed into $2 \mathrm{~cm}$ slabs typically tend to constitute FTL consignments compared to raw blocks to be processed into $1 \mathrm{~cm}$ slabs.

\subsubsection{Raw Dimension Stone Transformation}

The differences in texture, mineral composition, and other physical characteristics among different varieties of granites mean that processing times at the raw block transformation facilities tend to vary widely among different varieties for a given thickness. For the same reasons, allocated fixed cost (depending on the capacity of the plant) and variable costs (primarily, labor and material costs) also tend to vary depending on the variety and thickness of the dimension stone slab under process. For instance, granites higher in quartz content tend to be harder to cut and polish resulting in higher variable costs of processing compared to varieties with a lower quartz content. In addition, thicker slabs tend to require more consumables and labor for unit output.

\subsubsection{Outbound Transportation}

Outbound handling and transportation costs from a higher capacity processing facility location to domestic distribution centers/ seaports tend to be relatively cheaper compared to a lower capacity processing facility because of higher levels of mechanization, reduced labor costs, FTL consignments, and quantity-based discounts typically offered by Third-party Logistics (3PL) providers.

\subsection{Dimension Stone Supply Chain Network Design}

Availability of a particular variety of dimension stone is generally unique to a specific geographic region (sometimes just a few square miles in size) and to that extent responsiveness as a performance metric is generally not a very high priority order-winner because of the limited options a typical buyer will have to buy a specific variety of dimension stone. Because of this, supply chain efficiency is normally the key order-winner as it is directly related to the increase in supply chain surplus. As a result, supply chain network design becomes crucial particularly in the context of determining the processing facility location(s) and capacity allocation(s), in order to minimize the overall production and transportation costs. The key decision variables of interest are the optimal location(s) of the processing facilities, relevant capacity allocation(s), quantities of rough-cut granite stones to be transported from various quarry locations to the potential processing facility locations, and the quantities of processed granite slabs and tiles to be transported to different domestic distribution centers and seaport locations for subsequent export.

The authors have searched databases such as Proquest, ScienceDirect, EBSCOhost, Scopus, etc., with various combinations of key words relevant to the dimension stone industry, but have not found research literature that addresses the broad scope of issues of dimension stone network design and tactical planning with either MILP or any other methodologies. Based upon one of the author's professional experience operating within the granite industry in India, it is observed that the facility location decisions are typically based on their proximity either to the quarry locations or to major markets thereby missing the opportunity to holistically consider unit transportation cost for inbound and outbound from all possible supply locations (quarries) to demand locations (local markets and seaport locations). This results in sub-optimal costs for the supply chain through seeking local minima instead of global minima given the multitude of extant varieties in granite and numerous supply locations and destinations. The proposed MILP model captures most of these cost elements typically omitted in the ad-hoc approaches. As a result, the proposed MILP model identifies the optimal use of the available resources.

Another aspect of the ad-hoc methods is that processing decisions are normally based upon the availability of the material, type of the granite that was processed in the immediate past, delivery due dates etc. This results in suboptimal sized dimension stones being processed on different processing machinery thereby increasing the overall transformation costs. Through the holistic consideration of the relevant cost elements for different varieties of granite on different processing equipment the proposed LP based model 
allocates the blocks to the appropriate machinery in the most cost-efficient manner.

Potential methods that could be utilized for studying this problem include simulation, center of gravity location selection, and goal programming. In order to use simulation for the strategic supply chain network design, practitioners would need to extensively model the options and probable potential product demands, transportation costs, supply quantities, and processing costs. This would be a much more complex undertaking than developing a deterministic MILP model. The center of gravity location methodology is an excellent choice for selecting a single central location with a limited set of weighting factors, but it rapidly becomes more complex when identifying multiple locations of different capacities to support a supply chain network. Goal programming offers a relatively simple approach that can model problems with a large set of variables, constraints, and objectives. However, the determination of the appropriate weights for the set of objectives is not necessarily straightforward and can suffer from subjectivity among decision makers. Overall, a MILP approach can reflect the large sets of decision variables and constraints suitable for a network design or tactical planning model. Provided the problem is not extremely large, reasonable solutions can be obtained with comparatively easy to use spreadsheet modeling tools readily available to practitioners.

\section{MILP Model Formulation}

To make the model mathematically tractable, certain assumptions are made in the context of raw granite procurement and transformation without loss of generality. The supply potential of a dimension stone quarry is assumed to be very large compared to the typical annual demand for a given variety. However, there could be limitations on the quantity to be extracted in a given time period resulting from the quality of the rock deposit, degree of mechanization, and other relevant factors. It is assumed that all colors/shades can be processed at each of the potential plant locations; reciprocating gang saws at all the plants can cut slabs of any thickness; and each potential processing facility location can have either high capacity or low capacity. It is also assumed that information regarding the maximum processing capacity for each potential location (for both high/low capacities) of a specific variety of granite with specific thickness is known.

It should also be noted that unit inbound and outbound transportation costs, allocated fixed costs and variable costs for processing one square foot of granite of variety $v$ and thickness $t$ are considered to be different at high and low capacity plants primarily due to the labor and machinery related productivity differences and the allocated fixed and variable cost differential.

For example, because of higher levels of mechanization at high capacity plants, handling costs (considered as part of inbound and outbound transportation costs), and variable processing costs typically tend to be lower and allocated fixed costs typically tend to be higher (because of higher initial capital investments) compared to a low capacity plant. This consideration allows the model to mimic the reality more closely and is expected to be quite useful to practitioners to derive the fullest benefit of utilizing an optimization model that considers the cost elements at the granular level.

\section{Index Use:}

Variety/color/shade: $v \in \mathrm{V}$

Thickness of the sawn granite slab: $t \in \mathrm{T}$

Plant location: $f \in \mathrm{F}$

Plant location $f$ with high capacity: $\mathrm{f}_{\mathrm{H}} \in \mathrm{F}_{\mathrm{H}} ; \mathrm{F}_{\mathrm{H}} \subseteq \mathrm{F}$

Plant location $f$ with low capacity: $\mathrm{f}_{\mathrm{L}} \in \mathrm{F}_{\mathrm{L}} ; \mathrm{F}_{\mathrm{L}} \subseteq \mathrm{F}$

Dimension stone quarry location: $l \in \mathrm{L}$

Seaport location: $p \in \mathrm{P}$

International destination: $i \in \mathrm{I}$

Distribution center location: $c \in \mathrm{C}$

\section{Decision Variables:}

$\mathrm{QQ}_{\mathrm{vtlf}_{H}}=$ Quantity in 'square feet equivalent' of variety $v$ granite to be cut into a thickness $t$ slab at processing facility $f_{H}$ (processing facility $f$ with high capacity) shipped from the dimension stone quarry location $l$ during a given time period.

Typically, raw granite block transportation quantities are expressed either in cubic meters or metric tons, however to be consistent with processed granite units of measurement (usually in square feet) at downstream stages of the supply chain, a new term 'square feet equivalent', is introduced, which is simply the expected square footage of a particular thickness granite slab/tile from raw granite block shipments. This conversion will facilitate better understanding of the model presented and improve the mathematical tractability.

$\mathrm{QQ}_{\mathrm{vtlf}_{L}}=$ Quantity in 'square feet equivalent' of variety $v$ granite to be cut into a thickness $t$ slab at the processing facility $f_{L}$ (processing facility $f$ with low capacity) shipped from the dimension stone quarry location $l$ during a given time period.

$\mathrm{FP}_{\mathrm{vtf}_{H}}=$ Total quantity in square feet of variety $v$, thickness $t$ granite processed at $f_{H}$ during a given time period.

$\mathrm{FP}_{\mathrm{vtf}_{L}}=$ Total quantity in square feet of variety $v$, thickness $t$ granite processed at $f_{L}$ during a given time period.

$\mathrm{FD}_{\mathrm{vtf}_{H} \mathrm{c}}=$ Quantity in 'square feet' of variety $v$, thickness $t$ granite processed at $f_{H}$ and transported to the domestic distribution center $c$ during a given time period.

$\mathrm{FD}_{\mathrm{vtf}_{L} \mathrm{c}}=$ Quantity in 'square feet' of variety $v$, thickness $t$ granite processed at $f_{L}$ and transported to the domestic distribution center $c$ during a given time period.

$\mathrm{FE}_{\mathrm{vtf}_{H} \mathrm{pi}}=$ Quantity in 'square feet' of variety $v$, thickness $t$ granite processed at $f_{H}$ and transported to the seaport $p$ to be shipped to the international location $i$ during a given time period.

$\mathrm{FE}_{\mathrm{vtf}_{L} \mathrm{pi}}=$ Quantity in 'square feet' of variety $v$, thickness $t$ granite processed at $f_{L}$ and transported to the seaport $p$ to be shipped to the international location $i$ during a given time period.

$\mathrm{X}_{\mathrm{f}} \quad=$ Binary variable for locating a high capacity plant at location $f$

$\mathrm{Y}_{\mathrm{f}} \quad=$ Binary variable for locating a low capacity plant at location $f$ 


\section{Parameters:}

$\mathrm{QC}_{\mathrm{vtlf}_{H}}=$ Cost of transporting (\$) one 'square foot equivalent' of granite of variety $v$, thickness $t$ from quarry location $l$ to the processing facility $f_{H}$.

$\mathrm{QC}_{\mathrm{vtfl}_{L}}=$ Cost of transporting (\$) one 'square foot equivalent' of granite of variety $v$, thickness $t$ from quarry location $l$ to the processing facility $f_{L}$.

$\mathrm{FPC}_{\mathrm{vtf}_{H}} \quad=$ Estimated variable cost (\$) for processing one square foot of variety $v$, thickness $t$ granite slab at processing facility $f_{H}$.

$\mathrm{FPC}_{\mathrm{vtf}_{L}}=$ Estimated variable cost $(\$)$ for processing one square foot of variety $v$, thickness $t$ granite slab at processing facility $f_{L}$.

$\mathrm{FC}_{\mathrm{vtf}_{H}} \quad=$ Allocated fixed cost estimate $(\$)$ of processing one square foot of variety $v$, thickness $t$ granite slab at processing facility $f_{H}$.

$\mathrm{FC}_{\mathrm{vtf}_{L}} \quad=$ Allocated fixed cost estimate $(\$)$ of processing one square foot of variety $v$, thickness $t$ granite slab at processing facility $f_{L}$.

$\mathrm{FDC}_{\mathrm{vtf}_{H} c}=$ Transportation cost (\$) for one square foot of variety $v$, thickness $t$ granite processed at $f_{H}$ and transported to the domestic distribution center $c$.

$\mathrm{FDC}_{\mathrm{vff}_{L} c}=$ Transportation cost (\$) for one square foot of variety $v$, thickness $t$ granite processed at $f_{L}$ and transported to the domestic distribution center $c$.

$\mathrm{FEC}_{\mathrm{vtf}_{H} p i}=$ Transportation cost (\$) for one square foot of variety $v$, thickness $t$ granite processed at $f_{H}$ and transported to the seaport $p$ to be shipped to the international destination $i$.

$\mathrm{FEC}_{\mathrm{vtf}_{L} p i}=$ Transportation cost (\$) for one square foot of variety $v$, thickness $t$ granite processed at $f_{L}$ transported to the port $p$ to be shipped to the international destination $i$.

$\mathrm{C}\left(\mathrm{f}_{\mathrm{H}}\right)=$ Combined processing capacity available in square feet, for all possible slab varieties/thicknesses at a high capacity plant $f_{H}$ during a given time period.

$\mathrm{C}\left(\mathrm{f}_{\mathrm{L}}\right)=$ Combined processing capacity available in square feet, for all possible slab varieties/thicknesses at a low capacity plant $f_{L}$ during a given time period.

$\mathrm{EO}_{\mathrm{vti}}=$ Demand in square feet from an international location $i$ for granite slabs of variety $v$ and thickness $t$ during a given time period.

$\mathrm{DO}_{\mathrm{vtc}}=$ Demand in square feet from a domestic distribution center $c$ for granite slabs of variety $v$ and thickness $t$ during a given time period.

\section{Objective function:}

$\operatorname{MIN}\left\{\sum_{v} \sum_{t} \sum_{l} \sum_{f_{H}}\left(\mathrm{QQ}_{\mathrm{vufH}_{H}} * \mathrm{QC}_{\mathrm{vifH}_{H}}\right)+\right.$

$\sum_{v} \sum_{t} \sum_{l} \sum_{f_{L}}\left(\mathrm{QQ}_{\mathrm{vufl}_{L}} * \mathrm{QC}_{\mathrm{vifl}_{L}}\right)+$

$$
\begin{aligned}
& \sum_{v} \sum_{t} \sum_{f_{H}}\left(\mathrm{FP}_{\mathrm{vtf}_{H}} *\left(\mathrm{FC}_{\mathrm{vtf}_{H}}+\mathrm{FPC}_{\mathrm{vff}_{H}}\right)+\right. \\
& \sum_{v} \sum_{t} \sum_{f_{L}}\left(\mathrm{FP}_{\mathrm{vtf}_{L}} *\left(\mathrm{FC}_{\mathrm{vtf}_{L}}+\mathrm{FPC}_{\mathrm{vtf}_{L}}\right)+\right. \\
& \sum_{v} \sum_{t} \sum_{f_{H}} \sum_{p} \sum_{i}\left(\mathrm{FE}_{\mathrm{vtf}_{H} \mathrm{pi}^{*}} * \mathrm{FEC}_{\mathrm{vtf}_{H} p i}\right)+ \\
& \sum_{v} \sum_{t} \sum_{f_{L}} \sum_{p} \sum_{i}\left(\mathrm{FE}_{\mathrm{vtf}_{L} \mathrm{pi}^{2}} * \mathrm{FEC}_{\mathrm{vtf}_{L} p i}\right)+ \\
& \sum_{v} \sum_{t} \sum_{f_{H}} \sum_{c}\left(\mathrm{FD}_{\mathrm{vtf}_{H} \mathrm{c}} * \mathrm{FDC}_{\mathrm{vff}_{H} c}\right)+ \\
& \left.\sum_{v} \sum_{t} \sum_{f_{L}} \sum_{c}\left(\mathrm{FD}_{\mathrm{vef}_{L} \mathrm{c}} * \mathrm{FDC}_{\mathrm{vtf}_{L} c}\right)\right\}
\end{aligned}
$$

For each available granite variety and thickness combination, the objective function considers the relevant cost components that account for:

- Total cost of transportation for the quantity shipped (in square feet equivalent) from all the granite quarry locations to all the potential processing facility locations

- Total fixed and variable costs for all the potential processing facility locations with high and low capacities

- Total cost of transportation to meet the external demand considering all possible combinations of processing facility locations, seaport locations, and the relevant international destinations.

- Total cost of transportation to meet the domestic demand considering all possible combinations of processing facility locations and domestic distribution center locations.

\section{Inbound Transportation Constraints:}

$$
\begin{aligned}
& \mathrm{FP}_{\mathrm{vtf}_{H}} \leq \sum_{l} \mathrm{QQ}_{\mathrm{vtlf}_{H}} \forall v, t, f_{H} \\
& \mathrm{FP}_{\mathrm{vtf}_{L}} \leq \sum_{l} \mathrm{QQ}_{\mathrm{vtlf}_{L}}=\forall v, t, f_{L}
\end{aligned}
$$

The above constraints ensure that the quantity of granite processed at a processing facility with high or low capacity for each variety and thickness combination is upper bounded by the quantity supplied from all the quarries in a given time period.

\section{Processing Facility Capacity Constraints:}

$$
\begin{array}{ll}
\sum_{v} \sum_{t} \mathrm{FP}_{\mathrm{vtf}_{H}} \leq \mathrm{C}\left(\mathrm{f}_{\mathrm{H}}\right)^{*} \mathrm{X}_{\mathrm{f}} & \forall f_{H} \\
\sum_{v} \sum_{t} \mathrm{FP}_{\mathrm{vtf}_{L}} \leq \mathrm{C}\left(\mathrm{f}_{\mathrm{L}}\right)^{*} \mathrm{Y}_{\mathrm{f}} & \forall f_{L}
\end{array}
$$

$\mathrm{X}_{\mathrm{f}}+\mathrm{Y}_{\mathrm{f}} \leq 1 \quad \forall f$

Capacity constraints ensure that total quantity processed at a given location $f$ for all varieties $v$ and thicknesses $t$ is limited by the design capacity for a high/ or low capacity plant at that location; also, at each location, if a processing facility is going to be located it will be either a high or low capacity facility; if both $\mathrm{X}_{\mathrm{f}}$ and $\mathrm{Y}_{\mathrm{f}}$ are ' 0 ' in the optimal solution, $f$ will not be considered as a potential processing facility location. 
Outbound Transportation Constraints:

$$
\begin{aligned}
& \sum_{p} \sum_{i} \mathrm{FE}_{\mathrm{vtf}_{H} \mathrm{pi}}+ \\
& \sum_{c} \mathrm{FD}_{\mathrm{vtf}_{H} \mathrm{c}} \leq \mathrm{FP}_{\mathrm{vtf}_{H}} \forall v, t, f_{H}
\end{aligned}
$$

$$
\begin{aligned}
& \sum_{p} \sum_{i} \mathrm{FE}_{\mathrm{vtf}_{L} \mathrm{pi}^{+}} \\
& \sum_{c} \mathrm{FD}_{\mathrm{vtf}_{L} \mathrm{c}} \leq \mathrm{FP}_{\mathrm{vtf}_{L}} \quad \forall v, t, f_{L}
\end{aligned}
$$

The above constraints will ensure that for each variety $v$, thickness $t$ granite at a given processing facility location $\mathrm{f}$ (with high or low capacity), the quantity that is earmarked for export to all the international destinations $i \in I$ through all the seaports $\mathrm{p} \in \mathrm{P}$ plus the quantity destined for the domestic distribution centers $c \in C$ is upper bounded by the processed quantity available in a given time period.

$$
\begin{aligned}
& \sum_{f_{H}} \sum_{p} \mathrm{FE}_{\mathrm{vtf}_{H} \mathrm{pi}}+\sum_{f_{L}} \sum_{p} \mathrm{FE}_{\mathrm{vtf}_{L} \mathrm{pi}} \geq \mathrm{EO}_{\mathrm{vti}} \forall v, t, i \\
& \sum_{f_{H}} \mathrm{FD}_{\mathrm{vtf}_{H} \mathrm{c}}+\sum_{f_{L}} \mathrm{FD}_{\mathrm{vtf}_{L} \mathrm{c}} \geq \mathrm{DO}_{\mathrm{vtc}} \forall v, t, c
\end{aligned}
$$

Export demand constraints ensure that the total quantity of processed material (at all the facilities with high or low capacity) of variety $v$ and thickness $t$ that is earmarked for export to the international destination $i$ through all the seaports must be at least equal to the quantity demanded by the international destination $i$ for variety $v$ and thickness $t$ granite slabs in a given time period. Also, domestic demand constraints ensure that the total quantity of processed material (at all the facilities with high or low capacity) of variety $v$ and thickness $t$ that is earmarked for domestic consumption through the domestic distribution center $c$ must be at least equal to the quantity demanded by the region served by the distribution center $c$ for variety $v$ and thickness $t$ granite slabs in a given time period.

\section{Nonnegativity Constraints:}

All the decision variables are assumed to be nonnegative except $\mathrm{X}_{\mathrm{f}}$ and $\mathrm{Yf}$, which are binary in nature.

\subsection{Tactical Production Planning Model}

\subsubsection{Importance of Tactical Planning in the Dimension Stone Industry}

Once the supply chain network design has been finalized, medium-term tactical production plans and relevant supply chain network issues need to be addressed by considering firmed up orders and forecasts over the appropriate time horizon. Based on the confirmed and forecasted demand data, rough-cut capacity and master production plans will be prepared. However, tactical plan preparation in the dimension stone industry is relatively more complex, as one should take into consideration a multitude of scenarios that are unique to this industry.

For example, raw granite blocks do not all come in the same size; raw block dimensions vary widely depending on the type and quality of the sheet-rock deposit. The texture and hardness also differ based on the granularity and the composition of different types of granites. These issues mean that cutting, polishing, and trimming operations at the processing facilities must be scheduled carefully to minimize the cost of transformation operations. For example, reciprocating gang saws used in the cutting operation come in different sizes (such as large, medium and small) with cutting capacity varying between 7 to $3 \mathrm{~m}^{3}$ of the rough-cut dimension stone per single loading. Likewise, polishers come in different sizes, such as 4-Headed, 2-Headed etc., with different types of abrasive materials for smoothing the coarse surfaces of sawn blocks. Finally, trimmers also come in different capacities such as heavy duty, light duty, etc.

Even though any variety of granite can be processed on any combination of gang saw, polisher, and trimmer, it is typically not advisable if the goal is to minimize the cost of processing at the processing facility. It would be unnecessarily expensive to cut a small size raw granite block on a large size reciprocating gang saw because of the increased overhead expenses and the resulting sub-optimal utilization of the machinery. Depending on the granularity and hardness of the granite, certain types of polishers and trimmers are relatively cost effective if used on appropriate varieties of granites. Because of these reasons, the cost of processing will vary for cutting, polishing, and trimming operations depending on the type of granite being processed. The goal of the tactical plan development is to come up with an ideal configuration of the transformation processes that would result in an optimum production schedule subject to limitations on the capacities, demand for different varieties, and other relevant constraints.

Also, it should be noted that two independent models have been developed to address the two entirely different problems of facility location/capacity allocation through a network design model (sub-section 3.2) that is a one-time, long-term decision model and the development of a master production plan as a tactical planning problem (sub-section 3.3.2), which is a recurring decision making model with time horizons varying typically between 3 months to 2 years. However, we assume that expected processed quantities for different varieties of granite slabs as captured by decision variables $\mathrm{FP}_{\mathrm{vtf}_{H}}, \mathrm{FP}_{\mathrm{vtf}_{L}}$, etc., in the network design model presented in sub-section 3.2 will serve as the upper bounds of the $\mathrm{PQ}_{\mathrm{vtr}}, \mathrm{PQ}_{\mathrm{vto}}, \mathrm{PQ}_{\mathrm{vtm}}$ decision variables for individual processing operations as used in the model presented in subsection 3.3.2. We believe that adoption of the same set of relevant decision variables in both the models would unnecessarily increase the complexity of the strategic network design model by including individual processing step capacities rather than using a single broad measure of capacity. The tactical planning model presented in subsection 3.3.2 considers the capacities of individual processing steps and thus has a more detailed view for planning production. This approach of separate models may be especially beneficial to practitioners who want to use just the model presented in sub-section 3.3.2. Keeping the two models separate makes it easier for the tactical production 
planning model to be utilized by practitioners that are operating within an existing supply chain network rather than trying to design a new one.

\subsubsection{LP Model for Tactical Planning}

In the proposed LP model, it is assumed that the key operations that impact the capacity of the processing facility are cutting, polishing and trimming. It is further assumed that there are reciprocating gang saws, polishers, and trimmers available with different capacities to perform those tasks. For example, a hypothetical processing facility may have 3 reciprocating gang saws with large, medium, small capacities, two types of polishers (4-Head / 2-Head polishers), and two types of trimmers (heavy duty/ light duty trimmers). The goal is to determine the optimal configuration of the operations on the different machinery to minimize the cost of transformation in a given time period.

\section{Index Use:}

Variety/color/shade: $v \in \mathrm{V}$

Thickness of the sawn granite slab: $t \in \mathrm{T}$

Reciprocating gang saw: $\mathrm{r} \in \mathrm{R}$

Polisher: $o \in \mathrm{O}$

Trimmer: $m \in \mathrm{M}$

\section{Decision Variables:}

$\mathrm{PQ}_{\mathrm{vtr}}$ : Quantity in square feet equivalent of variety $v$, thickness $t$ granite processed on reciprocating gang saw $r$ in a given time-period.

$\mathrm{PQ}_{\text {vto }}$ : Quantity in square feet of variety $v$, thickness $t$ granite processed on polisher $o$ in a given timeperiod.

$\mathrm{PQ}_{\mathrm{vtm}}$ : Quantity in square feet of variety $v$, thickness $t$ granite processed on trimmer $m$ in a given timeperiod.

\section{Parameters:}

$\mathrm{PCT}_{\mathrm{vtr}}$ : Cost of processing one square foot equivalent of variety $v$, thickness $t$ granite on reciprocating gang saw $r$.

$\mathrm{PCT}_{\text {vto }}$ : Cost of processing one square foot of variety $v$, thickness $t$ granite on polisher $o$.

$\mathrm{PCT}_{\mathrm{vtm}}$ : Cost of processing one square foot of variety $v$, thickness $t$ granite on trimmer $m$.

$\mathrm{PCP}_{\mathrm{r}} \quad$ : Total processing capacity on reciprocating gang saw $r$ in a given time-period.

$\mathrm{PCP}_{\mathrm{o}} \quad$ : Total processing capacity on polisher $o$ in a given time-period.

$\mathrm{PCP}_{\mathrm{m}}$ : Total processing capacity on trimmer $m$ in a given time-period.

$\mathrm{D}_{\mathrm{vt}} \quad$ : Total demand for variety $v$ and thickness $t$ granite in a given time-period

\section{Objective function:}

$$
\begin{aligned}
& \operatorname{MIN}\left\{\sum_{v} \sum_{t} \sum_{r}\left(\mathrm{PQ}_{\mathrm{vtr}} * \mathrm{PCT}_{\mathrm{vtr}}\right)+\right. \\
& \sum \sum \sum\left(\mathrm{PQ}_{\mathrm{vto}} * \mathrm{PCT}_{\mathrm{vto}}\right)+ \\
& \left.\sum_{v} \sum_{t} \sum_{m}\left(\mathrm{PQ}_{\mathrm{vtm}} * \mathrm{PCT}_{\mathrm{vtm}}\right)\right\}
\end{aligned}
$$

The objective function minimizes the total cost of transformation operations on available processing machinery ( $r \in \mathrm{R}, \mathrm{o} \in \mathrm{O}$, and $\mathrm{m} \in \mathrm{M}$ ) for granite slabs/tiles of variety $v$ and thickness $t$ in a given time period. Even though we assumed any variety $v$ granite can be processed on any gang saw/polisher/trimmer combination that may not be practical at all the times due to technical and quality related issues. To allow this flexibility to practitioners, we considered each individual machine in a specific transformation operation (cutting/polishing/trimming) as a separate term in the above equation.

\section{Constraints:}

\section{Capacity constraints:}

$$
\begin{array}{ll}
\sum_{v} \sum_{t} \mathrm{PQ}_{\mathrm{vtr}} \leq \mathrm{PCP}_{\mathrm{r}} & \forall r \\
\sum_{v} \sum_{t} \mathrm{PQ}_{\mathrm{vto}} \leq \mathrm{PCP}_{\mathrm{o}} & \forall o \\
\sum_{v} \sum_{t} \mathrm{PQ}_{\mathrm{vtm}} \leq \mathrm{PCP}_{\mathrm{m}} & \forall m
\end{array}
$$

Capacity constraints for the processing machinery limit the total quantity of granite processed for all varieties $v$ and thicknesses $t$ in a given time period.

$$
\begin{array}{ll}
\sum_{r}^{\text {Demand constraints: }} \mathrm{PQ}_{\mathrm{vtr}} \geq \mathrm{D}_{\mathrm{vt}} & \forall v, t \\
\sum_{o} \mathrm{PQ}_{\mathrm{vto}} \geq \mathrm{D}_{\mathrm{vt}} & \forall v, t \\
\sum_{m} \mathrm{PQ}_{\mathrm{vtm}} \geq \mathrm{D}_{\mathrm{vt}} & \forall v, t
\end{array}
$$

Demand constraints ensure that for each variety $v$ and thickness $t$ the total amount of material processed on reciprocating gang-saws, polishers and trimmers is at least equal to the quantity demanded for a specific variety $v$ thickness $t$ combination in a given time period. Based on the demand profile data from the recent past for each variety $v$ thickness $t$ combination it is assumed that there is enough processing capacity to satisfy any future demand.

\section{Minimum quantity requirements:}

There may be scenarios in which granite varieties do not currently have sufficient demand to warrant production, but that management wants to promote to develop as niche varieties. In such cases, minimum quantities of production can be specified and included as demand constraints.

\section{Nonnegativity Constraints:}

All the decision variables are assumed to be nonnegative.

\section{NUMERICAL ANALYSIS}

In this section, two numerical examples are provided to illustrate the models presented in the previous section. For better illustration of the models presented in section 3 , two separate cases are presented in sub-sections 4.1 and 4.2 for the MILP based supply chain network design and the LP based tactical production planning problems for hypothetical entities Asian Granites Company and Alpha Tiles \& Slabs Inc., respectively. The company scenarios and data used in this section were simulated based upon one author's previous managerial experience within the dimension stone industry. To solve larger linear integer programming problems practitioners/researchers may use OpenSolver 2.9.0(opensolver.org) a free, open source software, which is 
compatible with Excel Solver among other options available. It is hoped that the practitioners will take advantage of the examples presented to help develop their own models for minimum cost strategic and tactical planning particularly when there are a multitude of varieties to choose from.

\subsection{Supply Chain Network Design as Capacitated Facility Location Problem}

This illustrative example envisions the Asian Granites Company located in South India primarily supplies two varieties of granites (called MR for Majestic Ruby and JB for Jet Black) as raw blocks (rough-cut stones) to local processing industries who in turn supply the processed granite to local and international markets to satisfy domestic and external demands. It is typical for a granite processing facility to process 10 or more varieties; however, we are considering only two varieties for the sake of mathematical tractability in this numerical analysis. The MR variety is procured from quarry locations in Raichur (RC) or Hospet (HP) both located in the state of Karnataka, and the JB variety is procured from either Ongole (ON) located in the state of Andhra Pradesh or Krishnagiri (KG) located in the state of Tamilnadu.

Asian Granites, previously a raw material supplier, is now considering further value-addition by expanding vertically into the raw block transformation stage of the granite supply chain. Asian Granites primarily wants to supply both the varieties as slabs that are either 1 or $2 \mathrm{~cm}$ in thickness. Based on the past demand data for these two varieties, Asian Granites estimates that there is an average demand of 600,000 sqft/year for the MR variety and an average demand of 300,000 sqft/year for the JB variety. Approximately two-thirds of the demand is domestic, and the rest is from the overseas markets for both varieties. There are two potential locations, Bangalore (BAN) and Chennai (CHE) where the management can construct and operate either a high capacity (HC) plant (720,000 sqft/year) or a low capacity (LC) plant (480,000 sqft/year). Domestic demand is routed through two distribution centers (DC) located in Hyderabad (HYD) and Bangalore (BAN) primarily to cater to the demand in the South India region. Overseas demand is primarily from the regions of Europe (EUR) and North America (NA) and met by shipping from the ports of either Mangalore (MAN) located on the West coast and/or Chennai (CHE) located on the East coast. Figure 3 depicts the dimension stone supply chain network for Asian Granites from raw material to market destination.

Forecasted annual demand information from both the local and international destinations have been developed as parameters for the numerical example. The simulated costs of transporting granite slabs from the quarry locations to the processing facilities; the costs to process a square foot of granite; and the costs to transport the granite from the processing facility to a location for outbound transport have also been developed for the example. The processing costs include allocated fixed cost and variable cost components for processing one square foot of granite at all the potential locations. For simplicity, all costs are denominated in U.S. dollars. Table 1A provides relevant data for the cost of inbound transportation from the granite quarry locations to potential processing facility locations broken down by the granite variety (MR vs. JB), the quarry location (RC, HP, $\mathrm{ON}$, or $\mathrm{KG})$, the slab thickness $(1 \mathrm{~cm}$ or $2 \mathrm{~cm})$, and which processing facility it is being sent to. Table $1 \mathrm{~A}$ also provides the fixed and variable costs of processing the slabs, the processing facility capacities, and the demand for each granite variety. The demand figures are structured as the total square footage per year for a variety and slab thickness regardless of the quarry it came from. The demand may be satisfied by solely shipping from either of the two quarries by variety or by a combination of shipments from both quarries that have that variety.

Table 1B shows the amounts of granite by variety, quarry location, and slab thickness that should be sent to specific processing facilities for the inbound transportation and thus how much should be processed at those locations in this portion of the minimum cost optimal solution.

Table 1C shows the remaining objective function coefficients and model parameters for the supply chain network design numerical example model. This table provides the costs of outbound transport from the processing facility to either a distribution center serving the domestic market (Bangalore or Hyderabad) or to a seaport (Mangalore or Chennai) for export overseas (to Europe or North America). The costs are specified by the granite variety, slab thickness, and the ultimate market destination. Table $1 \mathrm{C}$ also shows the product demand by variety, slab thickness, and domestic vs. international destination. The demand data are structured to reflect that export demand for Europe or North American may be met by solely shipping from either one of the seaports or a combination of shipments from both seaports.

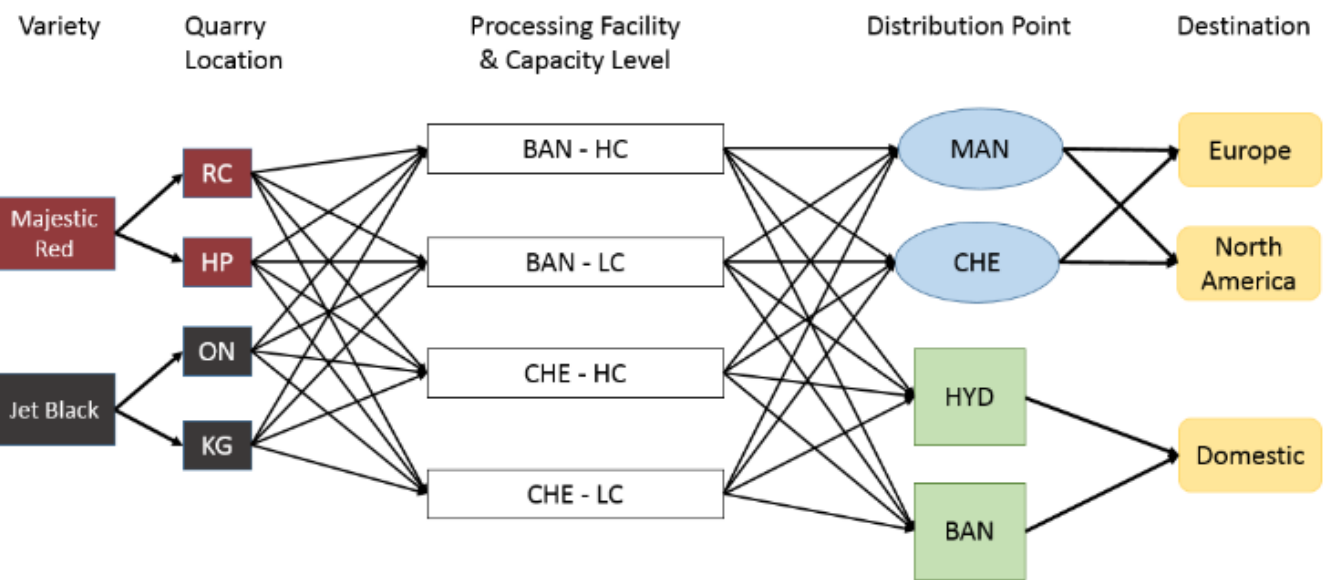

Figure 3. Asian granites co. dimension stone supply chain network 
Table 1A. Supply chain network design -objective function coefficients \& parameter values for inbound transportation and processing

\begin{tabular}{|c|c|c|c|c|c|c|c|c|c|}
\hline \multirow{3}{*}{$\begin{array}{c}\text { Variety, } \\
\text { Quarry, \& } \\
\text { Slab } \\
\text { Thickness }\end{array}$} & \multicolumn{8}{|c|}{ Processing Facility Location \& High vs. Low Cost } & \multirow{3}{*}{$\begin{array}{l}\text { Demand } \\
\text { (sqft/yr) }\end{array}$} \\
\hline & \multicolumn{4}{|c|}{$\begin{array}{l}\text { Cost of Transport from Quarry to } \\
\text { Processing Facility (\$/sqft) }\end{array}$} & \multicolumn{4}{|c|}{ Processing Cost by Location (\$/sqft) } & \\
\hline & $\begin{array}{l}\text { BAN } \\
\text { (HC) }\end{array}$ & $\begin{array}{l}\text { BAN } \\
\text { (LC) }\end{array}$ & $\begin{array}{l}\mathrm{CHE} \\
(\mathrm{HC})\end{array}$ & $\begin{array}{l}\text { CHE } \\
\text { (LC) }\end{array}$ & BAN (HC) & BAN (LC) & $\mathrm{CHE}(\mathrm{HC})$ & CHE (LC) & \\
\hline MR-RC-1cm & 0.80 & 1.00 & 1.20 & 1.40 & 2.40 & 3.00 & 2.50 & 3.10 & \multirow[t]{2}{*}{300,000} \\
\hline MR-HP-1cm & 0.60 & 0.80 & 1.00 & 1.20 & 2.40 & 3.00 & 2.50 & 3.10 & \\
\hline MR-RC-2cm & 0.70 & 0.90 & 1.10 & 1.30 & 2.00 & 2.50 & 2.00 & 1.80 & \multirow[t]{2}{*}{300,000} \\
\hline MR-HP-2cm & 0.50 & 0.70 & 0.90 & 1.10 & 2.00 & 2.50 & 2.00 & 1.80 & \\
\hline JB-ON-1cm & 1.20 & 1.30 & 1.30 & 1.40 & 3.20 & 2.80 & 3.30 & 2.90 & \multirow[t]{2}{*}{150,000} \\
\hline JB-KG-1cm & 1.05 & 1.15 & 1.15 & 1.25 & 3.20 & 2.80 & 3.30 & 2.90 & \\
\hline JB-ON-2cm & 1.10 & 1.20 & 1.20 & 1.30 & 2.70 & 2.30 & 2.80 & 2.40 & \multirow[t]{2}{*}{150,000} \\
\hline JB-KG-2cm & 0.95 & 1.05 & 1.05 & 1.15 & 2.70 & 2.30 & 2.80 & 2.40 & \\
\hline \multicolumn{5}{|c|}{ Processing Facility Capacity (sqft/yr) } & 720,000 & 480,000 & 720,000 & 480,000 & \\
\hline
\end{tabular}

Table 1B. Supply chain network design - optimal solution for inbound transportation and processing

\begin{tabular}{|c|c|c|c|c|c|c|c|c|c|}
\hline \multirow{3}{*}{$\begin{array}{c}\text { Variety, } \\
\text { Quarry, \& } \\
\text { Slab } \\
\text { Thickness }\end{array}$} & \multicolumn{8}{|c|}{ Processing Facility Location \& High vs. Low Cost } & \multirow{3}{*}{$\begin{array}{l}\text { Demand } \\
\text { (sqft/yr) }\end{array}$} \\
\hline & \multicolumn{4}{|c|}{$\begin{array}{l}\text { Quantity Transported from Quarry to } \\
\text { Processing Facility (sqft/yr) }\end{array}$} & \multicolumn{4}{|c|}{ Quantity Processed by Location (sqft/yr) } & \\
\hline & BAN (HC) & $\begin{array}{l}\text { BAN } \\
\text { (LC) }\end{array}$ & $\mathrm{CHE}(\mathrm{HC})$ & $\begin{array}{l}\mathrm{CHE} \\
\text { (LC) }\end{array}$ & BAN (HC) & BAN (LC) & CHE (HC) & CHE (LC) & \\
\hline MR-RC-1cm & 0 & 0 & 0 & 0 & 0 & 0 & 0 & 0 & \multirow[t]{2}{*}{300,000} \\
\hline MR-HP-1cm & 100,000 & 0 & 200,000 & 0 & 100,000 & 0 & 200,000 & 0 & \\
\hline MR-RC-2cm & 0 & 0 & 0 & 0 & 0 & 0 & 0 & 0 & \multirow[t]{2}{*}{300,000} \\
\hline MR-HP-2cm & 80,000 & 0 & 220,000 & 0 & 80,000 & 0 & 220,000 & 0 & \\
\hline JB-ON-1cm & 0 & 0 & 0 & 0 & 0 & 0 & 0 & 0 & \multirow[t]{2}{*}{150,000} \\
\hline JB-KG-1cm & 50,000 & 0 & 100,000 & 0 & 50,000 & 0 & 100,000 & 0 & \\
\hline JB-ON-2cm & 0 & 0 & 0 & 0 & 0 & 0 & 0 & 0 & \multirow[t]{2}{*}{150,000} \\
\hline JB-KG-2cm & 60,000 & 0 & 90,000 & 0 & 60,000 & 0 & 90,000 & 0 & \\
\hline \multicolumn{5}{|c|}{ Processing Facility Capacity (sqft/yr) } & 720,000 & 480,000 & 720,000 & 480,000 & \\
\hline
\end{tabular}

Table 1C. Supply chain network design - objective function coefficients \& parameter values for outbound transportation

\begin{tabular}{|c|c|c|c|c|c|}
\hline \multirow{3}{*}{$\begin{array}{l}\text { Variety, Size, \& Domestic/Export } \\
\text { Destination }\end{array}$} & \multicolumn{4}{|c|}{$\begin{array}{c}\text { Cost of Transport from Processing Facility Location to Distribution } \\
\text { Center/Seaport (\$/sqft) }\end{array}$} & \multirow{3}{*}{$\begin{array}{l}\text { Destination Demand } \\
\text { by Variety \& Size } \\
\text { (sqft/yr) }\end{array}$} \\
\hline & \multicolumn{2}{|c|}{ Bangalore (BAN) } & \multicolumn{2}{|c|}{ Chennai (CHE) } & \\
\hline & $\mathrm{HC}$ & LC & $\mathrm{HC}$ & LC & \\
\hline MR-1cm-BAN & 0.20 & 0.30 & 1.00 & 1.10 & 100,000 \\
\hline MR-1cm-HYD & 1.00 & 1.10 & 1.40 & 1.50 & 100,000 \\
\hline MR-1cm-MAN-EUR & 1.10 & 1.20 & 1.50 & 1.60 & 60,000 \\
\hline MR-1cm-CHE-EUR & 0.80 & 0.90 & 0.20 & 0.30 & \\
\hline MR-1cm-MAN-NA & 1.10 & 1.20 & 1.50 & 1.60 & 40,000 \\
\hline MR-1cm-CHE-NA & 0.80 & 0.90 & 0.20 & 0.30 & \\
\hline MR-2cm-BAN & 0.15 & 0.25 & 0.90 & 1.00 & 80,000 \\
\hline MR-2cm-HYD & 0.90 & 1.00 & 1.30 & 1.40 & 120,000 \\
\hline MR-2cm-MAN-EUR & 1.00 & 1.10 & 1.40 & 1.50 & 60,000 \\
\hline MR-2cm-CHE-EUR & 0.70 & 0.80 & 0.15 & 0.25 & \\
\hline MR-2cm-MAN-NA & 1.00 & 1.10 & 1.40 & 1.50 & 40,000 \\
\hline MR-2cm-CHE-NA & 0.70 & 0.80 & 0.15 & 0.25 & \\
\hline JB-1cm-BAN & 0.30 & 0.40 & 1.10 & 1.20 & 50,000 \\
\hline JB-1cm-HYD & 1.10 & 1.20 & 1.50 & 1.60 & 50,000 \\
\hline JB-1cm-MAN-EUR & 1.20 & 1.30 & 1.60 & 1.70 & 30,000 \\
\hline JB-1cm-CHE-EUR & 0.90 & 1.00 & 0.30 & 0.40 & \\
\hline JB-1cm-MAN-NA & 1.20 & 1.30 & 1.60 & 1.70 & 20,000 \\
\hline JB-1cm-CHE-NA & 0.90 & 1.00 & 0.30 & 0.40 & \\
\hline JB-2cm-BAN & 0.25 & 0.35 & 1.00 & 1.10 & 60,000 \\
\hline JB-2cm-HYD & 1.00 & 1.10 & 1.40 & 1.50 & 40,000 \\
\hline JB-2cm-MAN-EUR & 1.10 & 1.20 & 1.50 & 1.60 & 35,000 \\
\hline JB-2cm-CHE-EUR & 0.80 & 0.90 & 0.25 & 0.35 & \\
\hline JB-2cm-MAN-NA & 1.10 & 1.20 & 1.50 & 1.60 & 15,000 \\
\hline JB-2cm-CHE-NA & 0.80 & 0.90 & 0.25 & 0.35 & \\
\hline
\end{tabular}


Table 1D. Supply chain network design - optimal solution for outbound transportation

\begin{tabular}{|c|c|c|c|c|c|}
\hline \multirow{3}{*}{$\begin{array}{l}\text { Variety, Size, \& Domestic/Export } \\
\text { Destination }\end{array}$} & \multicolumn{4}{|c|}{$\begin{array}{l}\text { Quantity Transported from Processing Facility Location to Distribution } \\
\text { Center/Seaport (sqft/yr) }\end{array}$} & \multirow{3}{*}{$\begin{array}{l}\text { Destination Demand } \\
\text { by Variety \& Size } \\
\text { (sqft/yr) }\end{array}$} \\
\hline & \multicolumn{2}{|c|}{ Bangalore (BAN) } & \multicolumn{2}{|c|}{ Chennai (CHE) } & \\
\hline & $\mathrm{HC}$ & LC & $\mathrm{HC}$ & LC & \\
\hline MR-1cm-BAN & 100,000 & 0 & 0 & 0 & 100,000 \\
\hline MR-1cm-HYD & 0 & 0 & 100,000 & 0 & 100,000 \\
\hline MR-1cm-MAN-EUR & 0 & 0 & 0 & 0 & 60,000 \\
\hline MR-1cm-CHE-EUR & 0 & 0 & 60,000 & 0 & \\
\hline MR-1cm-MAN-NA & 0 & 0 & 0 & 0 & 40,000 \\
\hline MR-1cm-CHE-NA & 0 & 0 & 40,000 & 0 & \\
\hline MR-2cm-BAN & 80,000 & 0 & 0 & 0 & 80,000 \\
\hline MR-2cm-HYD & 0 & 0 & 120,000 & 0 & 120,000 \\
\hline MR-2cm-MAN-EUR & 0 & 0 & 0 & 0 & 60,000 \\
\hline MR-2cm-CHE-EUR & 0 & 0 & 60,000 & 0 & \\
\hline MR-2cm-MAN-NA & 0 & 0 & 0 & 0 & 40,000 \\
\hline MR-2cm-CHE-NA & 0 & 0 & 40,000 & 0 & \\
\hline JB-1cm-BAN & 50,000 & 0 & 0 & 0 & 50,000 \\
\hline JB-1cm-HYD & 0 & 0 & 50,000 & 0 & 50,000 \\
\hline JB-1cm-MAN-EUR & 0 & 0 & 0 & 0 & 30,000 \\
\hline JB-1cm-CHE-EUR & 0 & 0 & 30,000 & 0 & \\
\hline JB-1cm-MAN-NA & 0 & 0 & 0 & 0 & 20,000 \\
\hline JB-1cm-CHE-NA & 0 & 0 & 20,000 & 0 & \\
\hline JB-2cm-BAN & 60,000 & 0 & 0 & 0 & 60,000 \\
\hline JB-2cm-HYD & 0 & 0 & 40,000 & 0 & 40,000 \\
\hline JB-2cm-MAN-EUR & 0 & 0 & 0 & 0 & 35,000 \\
\hline JB-2cm-CHE-EUR & 0 & 0 & 35,000 & 0 & \\
\hline JB-2cm-MAN-NA & 0 & 0 & 0 & 0 & 15,000 \\
\hline JB-2cm-CHE-NA & 0 & 0 & 15,000 & 0 & \\
\hline
\end{tabular}

Table 1D shows the amounts of granite by variety, slab thickness, and ultimate market destination that should be sent from the processing facilities to the distribution centers and seaports in the minimum cost optimal solution.

The goal of the Asian Granites' management is to determine the processing facility location(s) and the relevant capacity allocations in an optimal fashion such that the total cost of production (fixed and variable costs) and transportation through the supply chain network are minimized. The optimal solution obtained for this numerical analysis recommends high capacity plants at both the potential locations (Bangalore and Chennai) with the total annual cost of processing and transportation coming to $\$ 3,674,500$, while satisfying all the supply, demand, and capacity related constraints.

Solver answer and sensitivity reports that are automatically generated provide valuable information for effective decision making for the practitioners. For example, the Solver answer report for the strategic network model indicates that only the capacity constraints at Chennai (HC) and Bangalore (HC) locations are non-binding and the rest of the constraints are all binding meaning that there are unused capacities at these plant locations. At Chennai (HC) out of the design capacity of 720,000 sqft/year, estimated internal \& external demand of 610,000 sqft/year will be satisfied resulting in an utilization rate of $84.72 \%$; assuming in allowances for maintenance and other down times to approximately $10 \%$ of the capacity, we can see that there is little scope for increasing the utilization at Chennai (HC). Whereas, at Bangalore (HC), out of the design capacity of $720,000 \mathrm{sqft} /$ year, estimated internal \& external demand of $290,000 \mathrm{sqft} /$ year will be satisfied resulting in a utilization rate of $40.27 \%$. Clearly there is significant scope at Bangalore (HC) for increasing the utilization of the existing processing capacity. Decision makers may explore avenues for additional markets not only for MR and JB varieties but may also promote other varieties aggressively through a tailored marketing strategy.

\subsection{Tactical Production Planning Model}

To facilitate the inclusion of a relatively larger number of granite varieties in this section as opposed to the limit of just two varieties (as in sub-section 4.1), we are considering a different hypothetical processing facility, Alpha Tiles \& Slabs Inc., to demonstrate the tactical model. Alpha Tiles \& Slabs Inc. specializes in the processing of a wide variety of granites into tiles and slabs, which are in demand both in the domestic and external markets. To develop the necessary tactical production plans in the medium term, various production processes need to be configured taking into consideration technical and market related limitations. For example, raw granite blocks come in different sizes varying in volume from 1.5 to $6.75 \mathrm{~m}^{3}$. They also differ in their mineral composition, texture, and hardness necessitating the requirement of different types of Maintenance, Repair, and Operations (MRO) consumables during their transformation. For example, reciprocating gang saws based on their basic frame size are suited to cut a particular average sized raw block at the lowest possible cost; if a relatively larger or smaller size raw block needs to be processed it costs more. Also, the polisher capacity (4- Headed, 2-Headed etc.) and the types of the abrasives used in polishing operations tend to vary depending on the size and type of granite being processed resulting in a cost differential for different varieties. For similar reasons, variable costs per square foot differ to process different varieties of granite on the trimming machines (Heavy duty, Light duty, etc.) 
Alpha Tiles \& Slabs Inc. has three reciprocating gang saws RGS1, RGS2 and RGS3 designed to process blocks of average size $6.75 \mathrm{~m}^{3}, 4 \mathrm{~m}^{3}$, and $1.75 \mathrm{~m}^{3}$ respectively at the lowest cost; typically, red varieties come in larger sizes and black varieties come in smaller sizes primarily because of the nature of rock deposits, inherent cracks, and other geological non-conformities. Alpha Tiles \& Slabs Inc. has average processing cost estimates for all the transformation operations per square foot of each variety at its facilities. The per square foot cost estimates by variety at its facilities, the monthly demand by variety, and the processing capacity per month at its facilities are shown in Table 2A. To simplify this illustrative example, only ten varieties are considered all of which have the same slab thickness. For example, Majestic Red, Robust Red, Ivory White, and Classic Quartz varieties usually come in large size ( $3 \mathrm{~m} \times 1.5 \mathrm{~m} \times 1.5 \mathrm{~m})$, and it is cheaper to process these varieties on RGS1, which is designed to process large sized blocks in a cost-efficient manner. It would be progressively more expensive to process these varieties on RGS2 or RGS3 as significant changes would be required to handle larger sized blocks. Management wants to promote Nebula as a niche variety with a minimum production quantity of $6,000 \mathrm{sqft} / \mathrm{month}$. Because of the highly variable demand for different varieties of granite month to month, one-month is considered as the planning horizon in this numerical example.

The Jet Black, Black Gold, and Nebula varieties typically come in small sizes because of a multitude of natural cracks and other structural deficiencies. Thus, it is more economical to process them on a 2-Head Polisher (compared to other varieties) since it is more suitable for processing sawn raw granite sheets of smaller surface area (such as $1.75 \mathrm{~m} \mathrm{x} 1 \mathrm{~m}$ ) and softer mineral composition. A 4Head Polisher, which is designed to process relatively moderate or larger size sawn granite sheets of harder mineral composition would not be suitable for the granite varieties typically cut into smaller sizes. The trimmer (Heavy duty and Light-duty) cost structure for processing different varieties in Table $2 \mathbf{A}$ can also be understood on similar lines.

The minimum cost optimal solution to this tactical planning numerical example is shown in Table 2B. Monthly production for each variety, capacity and utilization related details are also shown in Table 2B. Subject to the capacity constraints on each of the processing equipment (total processed quantity of all the varieties must be less than the available capacity in each time period) and the demand constraints (for each variety, total amount processed on all the equipment for each operation must at least equal the demand for that variety in each time period) optimal processing cost is found to be US\$439,970.

The Solver answer report indicates that only the capacity constraints for the processing machinery RGS1, RGS2, RGS3, 4HP, 2HP, HDT and LDT are not-binding and the rest of the constraints are all binding. It can be noted that utilization rates ( $\%$ of processing capacity used) are $60.56 \%$, $61.9 \%, 80 \%, 87 \%, 24 \%, 99.43 \% \& 20.57 \%$ respectively for RGS1, RGS2, RGS3, 4HP, 2HP, HDT and LDT indicate that there are opportunities to be explored for improving the utilization of $2 \mathrm{HP}$ and LDT processing equipment with their current low rates of utilization at $24 \%$ and $20.57 \%$. This means management can explore ways to increase the demand for medium and small sized varieties such as Ocean Blue, Jet Black etc., as they are relatively cheaper to process on these equipment; such an approach will also increase the utilization of RGS2 and RGS3 as well, which are currently at $61.9 \%$ and $80 \%$ rates of utilization. Also, HDT utilization at $99.43 \%$ calls for augmenting additional capacity, if possible, to be able to meet demand spikes and to provide managerial flexibility in planning for maintenance and allowing for downtime. In addition, the 'Range of Optimality' table in the sensitivity report indicates that for example, for Majestic Ruby (MR) variety, which is to be totally processed on RGS1 for cutting operation at the rate of US\$1.1/sqft in optimal solution will not change even if the cost of cutting goes up by US\$0.34 or comes down by US\$1.10 giving additional flexibility for managers in consumables purchasing decisions.

Also, it can be learned from the sensitivity report that the Jet Black (JB) variety trimmer operation related constraint has the lowest shadow price of US\$0.16 among all the constraints indicating that overall processing costs will increase at the slowest rate for satisfying each additional unit of demand of this variety on the trimming operation. This is very useful information for decision makers employing such a model to look closely at their current marketing strategy for increasing demand for different varieties.

\section{DISCUSSION}

In this research, probably for the first time, a dimension stone supply chain is presented in considerable detail along with a MILP based strategic network design model and LP based tactical production planning model. In addition to addressing some of the unique challenges, as relevant to the processing of granite such as the multitude of variety available, variable processing requirements etc., explanatory numerical analyses are also presented primarily to facilitate the usage of the proposed models by dimension stone industry practitioners in small and medium scale business entities. The lack of research literature on dimension stone industry, hopefully, makes this research fill the gap in the application of quantitative models in this major industry. Also, compared to the research done in closely related industries, this research has made certain unique contributions as described below.

Compared to research papers that consider upstream operational aspects such as McKenzie et al. (2008), which deals with the optimal movement of the mining related equipment, Newman et al. (2010) that reviews the mathematical models in the mine planning and design, equipment selection etc., and research that focuses upon the downstream activities such as the marketing perspective of processed dimension stones (Careddu et al. 2017) our models exclusively address the post quarrying aspects of dimension stone supply chain such as inbound transportation of the quarried dimension stone to the processing facility, material transformation, outbound transportation of the processed material etc. 
Table 2A. Tactical planning in dimension stone industry - objective function coefficients \& parameter values

\begin{tabular}{|c|c|c|c|c|c|c|c|c|}
\hline \multirow[t]{2}{*}{ Granite Variety } & \multicolumn{3}{|c|}{$\begin{array}{l}\text { Cutting Cost } \\
\text { (\$/sqft) }\end{array}$} & \multicolumn{2}{|c|}{$\begin{array}{l}\text { Polishing Cost } \\
\text { (\$/sqft) }\end{array}$} & \multicolumn{2}{|c|}{$\begin{array}{c}\text { Trimming Cost } \\
\text { (\$/sqft) }\end{array}$} & \multirow[t]{2}{*}{$\begin{array}{l}\text { Demand } \\
\text { (sqft/month) }\end{array}$} \\
\hline & RGS1 & RGS2 & RGS3 & 4HP & $2 \mathrm{HP}$ & HDT & LDT & \\
\hline Majestic Ruby (MR) & 1.10 & 1.44 & 1.64 & 0.72 & 0.90 & 0.24 & 0.30 & 65,000 \\
\hline Robust Red (RR) & 1.20 & 1.54 & 1.74 & 0.82 & 1.00 & 0.34 & 0.40 & 35,000 \\
\hline Ivory White (IW) & 1.30 & 1.64 & 1.84 & 0.92 & 1.10 & 0.44 & 0.50 & 5,000 \\
\hline Classic Quartz (CQ) & 1.40 & 1.74 & 1.94 & 1.02 & 1.20 & 0.54 & 0.60 & 4,000 \\
\hline Ocean Blue (OB) & 1.30 & 0.96 & 1.50 & 0.62 & 0.80 & 0.19 & 0.25 & 25,000 \\
\hline Emerald Green (EG) & 1.40 & 1.06 & 1.60 & 0.72 & 0.90 & 0.29 & 0.35 & 30,000 \\
\hline Cheetah Spots (CS) & 1.50 & 1.16 & 1.70 & 0.82 & 1.00 & 0.39 & 0.45 & 10,000 \\
\hline Jet Black (JB) & 1.44 & 1.24 & 0.90 & 0.75 & 0.57 & 0.22 & 0.16 & 10,000 \\
\hline Black Gold (BG) & 1.54 & 1.34 & 1.00 & 0.85 & 0.67 & 0.32 & 0.26 & 20,000 \\
\hline Nebula (NE) & 1.64 & 1.44 & 1.10 & 0.95 & 0.77 & 0.42 & 0.36 & 6,000 \\
\hline $\begin{array}{l}\text { Process Capacity } \\
\text { (sqft/month) }\end{array}$ & 180,000 & 105,000 & 45,000 & 200,000 & 150,000 & 175,000 & 175,000 & \\
\hline
\end{tabular}

Table 2B. Tactical planning in dimension stone industry - optimal solution

\begin{tabular}{|c|c|c|c|c|c|c|c|c|}
\hline \multirow[t]{2}{*}{ Granite Variety } & \multicolumn{3}{|c|}{$\begin{array}{l}\text { Cutting Process Assignment } \\
\text { (sqft/month) }\end{array}$} & \multicolumn{2}{|c|}{$\begin{array}{c}\text { Polishing Process } \\
\text { Assignment } \\
\text { (sqft/month) }\end{array}$} & \multicolumn{2}{|c|}{$\begin{array}{c}\text { Trimming Process } \\
\text { Assignment (sqft/month) }\end{array}$} & \multirow[t]{2}{*}{$\begin{array}{l}\text { Production } \\
\text { (sqft/month) }\end{array}$} \\
\hline & RGS1 & RGS2 & RGS3 & 4HP & $2 \mathrm{HP}$ & HDT & LDT & \\
\hline Majestic Ruby (MR) & 65,000 & 0 & 0 & 65,000 & 0 & 65,000 & 0 & 65,000 \\
\hline Robust Red (RR) & 35,000 & 0 & 0 & 35,000 & 0 & 35,000 & 0 & 35,000 \\
\hline Ivory White (IW) & 5,000 & 0 & 0 & 5,000 & 0 & 5,000 & 0 & 5,000 \\
\hline Classic Quartz (CQ) & 4,000 & 0 & 0 & 4,000 & 0 & 4,000 & 0 & 4,000 \\
\hline Ocean Blue (OB) & 0 & 25,000 & 0 & 25,000 & 0 & 25,000 & 0 & 25,000 \\
\hline Emerald Green (EG) & 0 & 30,000 & 0 & 30,000 & 0 & 30,000 & 0 & 30,000 \\
\hline Cheetah Spots (CS) & 0 & 10,000 & 0 & 10,000 & 0 & 10,000 & 0 & 10,000 \\
\hline Jet Black (JB) & 0 & 0 & 10,000 & 0 & 10,000 & 0 & 10,000 & 10,000 \\
\hline Black Gold (BG) & 0 & 0 & 20,000 & 0 & 20,000 & 0 & 20,000 & 20,000 \\
\hline Nebula (NE) & 0 & 0 & 6,000 & 0 & 6,000 & 0 & 6,000 & 6,000 \\
\hline $\begin{array}{l}\text { Process Capacity Used } \\
\text { (sqft/month) }\end{array}$ & 109,000 & 65,000 & 36,000 & 174,000 & 36,000 & 174,000 & 36,000 & \\
\hline $\begin{array}{l}\% \text { Utilization of Process } \\
\text { Capacity }\end{array}$ & $60.56 \%$ & $61.90 \%$ & $80.00 \%$ & $87.00 \%$ & $24.00 \%$ & $99.43 \%$ & $20.57 \%$ & \\
\hline
\end{tabular}

In the context of determining the processing facility locations in similar industries, Safari et al. (2010) and Bakhtavar et al. (2017) primarily focus on the optimal location of the processing facility close to the mining area based on relevant local factors. Whereas, in our research we adopted a holistic approach for determining the optimal location of the processing facility considering the dimension stone quarry locations, transformation costs, and potential market locations, which mimics reality closely in developing countries.

In the context of model development, Kallrath (2002) and Corsano and Montagna (2011) consider strategic and operational planning decisions simultaneously. Whereas, we have developed two independent models dealing with strategic network design and tactical production planning separately considering the multi-dimensional complexity that is typical in dimension stone industry. This approach will also facilitate the independent use of these separate models by the practitioners as per their needs.

Holistic consideration of raw granite supply sources and demand locations for determining the processing facility location(s) in this research would hopefully dissuade industry practitioners from considering just the proximity either to the granite quarry locations or the major demand centers as the key criterion, which typically results in suboptimal supply chain costs. Tactical production planning model presented in this research, hopefully would help the industry practitioners to question the rationale in matching the processing equipment capacity to the raw block size while making tactical planning decisions.

\section{CONCLUSIONS}

Until now, the dimension stone industry has not benefited in a significant manner from the advancement of linear programming techniques and other advanced operations research models. Dimension stone supply chains, from the quarrying stage to the final consumer stage pose certain unique challenges, compared to other mineral commodity supply chains. A multitude of options in each dimension stone variety, coupled with unique processing requirements for specific varieties depending on their physical characteristics and the complexity of logistics operations make dimension stone supply chains ideal candidates for the application of linear programming models. This research is the first to describe the dimension stone supply chains in considerable detail and to develop theoretical models for capacitated facility location at the strategic level, and supply chain production planning at the tactical level. Application of the proposed theoretical models is also illustrated in considerable detail through the numerical examples, primarily to benefit industry practitioners. While a granite supply chain located in a developing nation is the setting chosen to explore various details throughout the paper, the models can easily be extended to any other global dimension stone supply chain 
network. With minor modifications, the proposed models can also be used in the ceramic tile industry for strategic and tactical planning. The proposed models for strategic and tactical planning are scalable, and additional stages (such as a retailer network) can be added if desired to make the model even more realistic. The proposed optimization models demonstrate through the demand profile and process related constraints that some notions currently in vogue such as matching block sizes to the processing equipment capacity would typically result in sub-optimal total costs.

Another novel feature of this model is the independent consideration of unit inbound and outbound transportation costs, allocated fixed costs and variable costs for processing one square foot of granite of variety $v$ and thickness $t$ at high and low capacity plants. These considerations at the granular level differentiate this model from other standard network design models and industry practitioners may find the proposed model more practical and appealing.

Future extensions of the models presented in this research could include considering stochastic demand and replenishment lead-times at different stages and consequently relevant implications for the safety-stock to be maintained at those stages. For such stochastic models, a simulation-based approach can be developed to compare various scenarios in the development of an appropriate strategic supply chain network model. Another option is developing relevant non-linear optimization models to arrive at the optimal cost estimations. Also, it is not uncommon to process different types of dimension stones in the same processing facility. The proposed model can be used either as is or with slight modifications to examine processing different types of stones as long as the stones' physical characteristics such as the hardness, mineral composition, and texture are sufficiently similar. Additional flexibility can be modeled for facilities that use different types of processing equipment (such as wire cutting, water jet cutting, automatic line polisher, etc.) with their own technical limitations. The differences in processing equipment can be accommodated in the presented framework with the addition of suitable constraints to determine whether particular varieties can be processed on particular types of processors.

Finally, it is earnestly hoped that practitioners will find the proposed models useful in reducing dimension stone supply chain network costs at both the strategic and tactical levels.

\section{REFERENCES}

Arntzen, B.C., Brown, G.G., Harrison, T.P., and Trafton, L.L. (1995). Global Supply Chain Management at Digital Equipment Corporation. Interfaces 25(1), pp. 69-93.

Babazadeh, R., Razmi, J., and Ghodsi, R. (2013). Facility location in responsive and flexible supply chain network design (SCND) considering outsourcing. International Journal of Operational Research 17(3), pp. 295-310.

Bakhtavar, E., Khademi, D., \& Mikaeil, R. (2017). The use of fuzzy-weighted binary integer goal programming to select the optimum site for a central processing plant. Journal of the Southern African Institute of Mining and Metallurgy, 117(5), pp. 505-510.

Baud-Lavigne, B., Agard, B., and Penz, B. (2016). Simultaneous product family and supply chain design: An optimization approach. International Journal of Production Economics 174, pp. 111-118.
Boujelben, M.K., Gicquel, C., and Minoux, M. (2016). A MILP model and heuristic approach for facility location under multiple operational constraints. Computers \& Industrial Engineering 98, pp. 446-461.

Careddu, N., Siotto, G., and Marras, G. (2017). The crisis of granite and the success of marble: errors and market strategies. The Sardinian case. Resources Policy 52, pp. 273-276.

Corsano, G., and Montagna, J.M. (2011). Mathematical modeling for simultaneous design of plants and supply chain in the batch process industry. Computers and Chemical Engineering 35, pp. 149-164.

Etemadnia, H., Goetz, S.J., Canning, P., and Tavallali, M.S. (2015). Optimal wholesale facilities location within the fruit and vegetables supply chain with bimodal transportation options: An LP-MIP heuristic approach. European Journal of Operational Research 244, pp. 648-661.

Fattahi, M., Mahootchi, M., and Moattar Husseini, S.M. (2016). Integrated strategic and tactical supply chain planning with price-sensitive demands. Annals of Operations Research 242(2), pp. 423-456.

Goodfellow, R. \& Dimitrakopoulos, R., (2017). Simultaneous Stochastic Optimization of Mining Complexes and Mineral Value Chains, Math Geosci 49, pp. 341-360.

Gumus, A.T., Guneri, A.F., and Keles, S. (2009). Supply chain network design using an integrated neuro-fuzzy and MILP approach: A comparative design study. Expert Systems with Applications 36, pp. 12570-12577.

Government of India, Ministry of Mines, Indian Bureau of Mines. (2014). "Granite" Indian Minerals Yearbook, 53 ${ }^{\text {rd }}$ Edition (Advance Release). Accessed September 11, 2017. http://ibm.nic.in/writereaddata/files/12182015100757IMYB2014_Granite (Adv).pdf

Kallrath, J. (2002). Combined strategic and operational planning An MILP success story in chemical industry. OR Spectrum 24(3), pp. 315-341.

Khan, H., and Wisner, J.D. (2019). Supply Chain Integration, Learning, and Agility: Effects on Performance. Operations and Supply Chain Management 12(1), pp. 14-23.

Korpela, J., and Lehmusvaara, A. (1999). A customer oriented approach to warehouse network evaluation and design. International Journal of Production Economics 59, pp. 135146.

Malek, I., and Rahim, A.R.A. (2017). Supply Chain Material Management Framework in Assemble to Orders (ATO). Operations and Supply Chain Management 10(1), pp. 35-46.

McKenzie, P., Newman, A.M., and Tenorio, L. (2008). Front Range Aggregates Optimizes Feeder Movements at its Quarry. Interfaces 38(6), pp. 436-447.

Newman, A.M., Rubio, E., Caro, R., Weintraub, A., and Eurek, K. (2010). A Review of Operations Research in Mine Planning. Interfaces 40(3), pp. 222-245.

Sabzevari Zadeh, A., Sahraeian, R. \& Homayouni, S.M. (2014), A dynamic multi-commodity inventory and facility location problem in steel supply chain network design. The International Journal of Advanced Manufacturing Technology 70 (5-8), pp. 1267-1282.

Safari, M., Ataei, M., Khalokakaie, R., \& Karamokzian, M. (2010). Mineral processing plant location using the analytic hierarchy process - a case study: the Sangan iron ore mine (phase 1). Mining Science and Technology (China), 20(5), pp. 691-695.

Saetta, S. and Tiacci, L. (2005). Lean simulation as a tool for the management of supply chain in the sector of natural stones. Summer Computer Simulation Conference 2005, SCSC 2005 , pp. 29-34.

Sharifzadeh, M., Garcia, M.C., and Shah, N. (2015). Supply chain network design and operation: Systematic decision-making for centralized, distributed, and mobile biofuel production using mixed integer linear programming (MILP) under uncertainty. Biomass and Bioenergy 81, pp. 401-414. 
Thanh, P.N., Bostel, N., and Peton, O. (2008). A dynamic model for facility location in the design of complex supply chains. International Journal of Production Economics 113, pp. 678693.

Tsiakis, P., and Papageorgiou, L.G. (2008). Optimal production allocation and distribution supply chain networks. International Journal of Production Economics 111, pp. 468483.
USGS (U.S. Geological Survey). (2017). Dimension Stone Statistics and Information. Accessed Feb 14, 2017 https://minerals.usgs.gov/minerals/pubs/commodity/stone_di mension/

USGS (U.S. Geological Survey). (2016). USGS Mineral Commodity Summaries: Stone (Dimension). Accessed Feb 15 , 2017. https://minerals.usgs.gov/minerals/pubs/commodity/stone_di mension/mcs-2016-stond.pdf

Dr. Gangaraju Vanteddu is currently serving as a Professor of Quantitative Methods at the Harrison College of Business and Computing, Southeast Missouri State University. He holds the B.Tech in Civil Engineering (S.V. University College of Engineering, Tirupati, India), M.Tech in Quality, Reliability and Operations Research ( Indian Statistical Institute, Kolkata, India), and Ph.D. in Industrial Engineering (Wayne State University, Detroit, USA). Dr. Vanteddu also currently maintains the Certified Supply Chain Professional (CSCP) certification from the APICS and Certified Quality Engineer (CQE) \& Certified Reliability Engineer (CRE) certifications from the ASQ. Dr. Vanteddu has published in reputed journals such as International Journal of Production Economics, International Journal of Production Research etc., and presented his research work at a number of national/international conferences. His primary research interests are in the area of supply chain management, applied statistics and six sigma quality.

Dr. Gillian M. Nicholls is an Associate Professor of Quantitative Methods at Southeast Missouri State University. Her research interests are in applying statistical analysis and optimization to supply chain management, transportation management, and engineering education. She holds the B.S. in Industrial Engineering (Lehigh University), Masters in Business Administration (Penn State University), M.S. in Industrial Engineering (University of Pittsburgh.), and Ph.D. in Industrial Engineering (University of Pittsburgh). Prior to entering academia, Dr. Nicholls was a practicing industrial engineer in the freight transportation industry. 